\title{
Implementación de la farmacogenética en la práctica clínica: hacia las estrategias de genotipado anticipado
}

\author{
Alberto M. Borobia ${ }^{1}$, Antonio J. Carcas Sansuán ${ }^{1^{*}}$ \\ ${ }^{1}$ Servicio de Farmacología Clínica. Hospital Universitario La Paz - IdiPAZ. Departamento de Farmacología y Terapéutica. Facultad de Medicina. \\ Universidad Autónoma de Madrid, Madrid, España. \\ *Autor de correspondencia: Antonio J. Carcas antonio.carcas@uam.es
}

Palabras claves: Farmacogenética, implementación, genotipado anticipado.

Recibido 23 de mayo, 2016; Aceptado 28 de mayo, 2016; Publicado 02 de junio, 2016.

Copyright: (c) 2016 Authors. This is an open-access article distributed under the terms of the Creative Commons Attribution License, which permits unrestricted use, distribution, and reproduction in any medium, provided the original author and source are credited. Editor: Jesús Frías Iniesta.

Citar como: Borobia AM., Carcas AJ., Implementación de la farmacogenética en la práctica clínica: hacia las estrategias de genotipado anticipado. IBJ Clinical Pharmacology 2016 1(1):e0002.

Fuentes de financiación: El autor declara que no hubo o ha habido subvención(es) involucrada(s) en el apoyo de este trabajo.

Conflictos de intereses: Antonio J. Carcas Sansuán es el fundador y editor jefe de Iberoamerican Journals (IBJ). Alberto M. Borobia es editor ejecutivo de IBJ Clinical Pharmacology. 
Los importantes avances realizados en los últimos años en el campo de la genética y la genómica han abierto la puerta a conocimientos y técnicas con un gran potencial de mejorar el diagnóstico y tratamiento de las enfermedades, dando paso al concepto de medicina personalizada. En lo que se refiere al tratamiento farmacológico estos conocimientos prometen dar un importante impulso a la individualización de la farmacoterapia: "el fármaco más adecuado a la dosis apropiada al paciente adecuado". El desarrollo de las técnicas genéticas en los últimos lustros ha permitido dar un salto cualitativo y cuantitativo en la investigación, ha impulsado el crecimiento de la literatura a un ritmo exponencial y ha generado un enorme crecimiento en el conocimiento de los factores genéticos que permiten explicar una parte, muy relevante en algunos casos, de la variabilidad en la respuesta a los fármacos. Esto ha permitido que la farmacogenética y la farmacogenómica se hayan establecido como disciplinas de gran importancia en el conocimiento médico. El efecto terapéutico de un fármaco va a depender de un largo camino que involucra procesos farmacocinéticos y farmacodinámicos, que a su vez pueden estar regulados por otros sistemas fisiológicos o patológicos. En todos estos procesos intervienen proteínas, enzimas, receptores, transportadores, entre otros, cuya expresión está codificada genéticamente. Estos genes pueden presentar variantes que pueden codificar productos con diferente grado de actividad o función, lo que en definitiva afectará el grado de actividad del fármaco (en términos de eficacia o de seguridad). Atendiendo a este esquema parece claro que las variaciones genéticas podrían constituirse en marcadores que nos permitirán predecir la respuesta farmacológica según estén o no presentes y su interrelación con otros marcadores.

En los últimos años diversos grupos y organismos han publicado sistemas de evaluación de las evidencias, guías, revisiones sistemáticas y evaluaciones para diferentes fármacos o patologías. Varios consorcios a nivel internacional pretenden compilar y evaluar la ingente cantidad de información farmacogenética existente, así como orientar a la comunidad médica sobre la utilización de esta información. Dentro de estos consorcios se encuentra "The Pharmacogenomics Knowledge Base (PharmGKB)", que recopila y ordena información farmacogenética basándose en un sistema de pirámide de conocimiento con varios niveles de evidencia. Es la primera base de datos "post-genómica" y actualmente incluye información propia y de otros consorcios, como "The Clinical Pharmacogenetics Implementation Consortium (CPIC)" que elabora guías de aplicación clínica de la farmacogenética que son publicadas tras revisión por pares en la revista Clinical Pharmacology and Therapeutics (1). La tabla 1, de acuerdo a PharmGKB, señala los fármacos para los que existe una recomendación por parte del Clinical

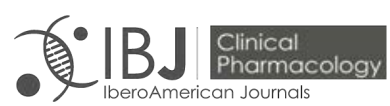

Pharmacogenetics Implementation Consortium (CPIC), el Dutch Pharmacogenetics Working Group (DPWG) o ambos.

También las agencias reguladoras de medicamentos, tanto la Food and Drug Administration (FDA) con la Agencia Europea de Medicamentos (EMA), incluyen información farmacogenética en las fichas técnicas de ciertos medicamentos. Así, podemos encontrarnos desde mera información sobre la relación genotipo-fenotipo ("Informative PGx") hasta advertencias que obligan a realizar determinados biomarcadores antes del inicio de un tratamiento ("Genetic testing required"). Esta información, así como el número de fármacos que la incluyen, es heterogénea entre las distintas agencias reguladoras, tal como se recoge en la página "Drug Labels" de PharmGKB.

Dentro de esta corriente de desarrollo de la farmacogenética se han detectado importantes barreras para la implementación y aplicación de la información farmacogenética en la práctica clínica habitual. Aunque no faltan ejemplos de aplicación rutinaria de esta información (2-7), lo cierto es que la gran mayoría de la información disponible no se aplica de manera generalizada. Las razones detectadas en la literatura para este estado de cosas incluyen la falta de acuerdo sobre las evidencias necesarias para implementar un biomarcador, la falta de formación en las técnicas farmacogenéticas y su interpretación y las limitaciones presupuestarias y organizativas necesarias dentro de los sistemas sanitarios.

Hasta el momento se han realizado diversas aproximaciones a la implementación de la farmacogenética en la práctica clínica:

A. Modelo caso-a-caso. En este modelo la decisión de ordenar una prueba genética es individualizada y se basa en la decisión de utilizar un fármaco influido por una o varias variaciones genéticas específicas. Una ventaja es que el resultado de la prueba genética va ser aplicada, ya que la decisión de ordenar la prueba genética está vinculada a la prescripción y es utilizada como una covariable junto con otras características del paciente en el manejo del fármaco. El mayor inconveniente es el coste de las determinaciones y la poca rapidez para estar disponible, lo que lo hace impracticable con frecuencia. Aunque se han desarrollado determinaciones para su realización "a pie de cama", esta aproximación está limitada a unos pocos casos (8).

B. Modelo de genotipado anticipado (9). Esta alternativa supone la obtención de la información genética at initio, de manera que estaría disponible cuando el paciente es evaluado y tratado. Actualmente el desarrollo de "arrays" permite determinar un número elevado de mutaciones cuyo impacto en la terapéutica es 
seguro o probable, dada la información disponible. Esta técnica es factible para cada paciente que entra en el sistema de atención de salud debido a su bajo coste y la información estaría disponible antes de cualquier decisión de prescripción. Una aproximación de este tipo, mediante el desarrollo de un "Array" con variantes de 225 genes se ha puesto en marcha en el St. Jude's Hospital (10) y otros autores han desarrollado proyectos similares de genotipado anticipado (11,12). Más recientemente esta aproximación ha dado un paso más y un grupo de USA ha planteado el proyecto eMERGE-PGx (13), un proyecto colaborativo de farmacogenotipado anticipado integrado en la historia clínica electrónica. Nuestro grupo (Hospital Universitario $\mathrm{La} \mathrm{Paz}$ ) ha puesto en marcha una estrategia similar gracias al desarrollo de un "Array" (PharmArray®) para la determinación de 192 variantes en 57 genes y que se utiliza de manera rutinaria para algunos casos determinados (Azatioprina/6-MercaptopurinaTPMT,Acenocumarol-CYP2C9/VKORC1/APOE/ CYP4F2, Anti-VHC-IL28b,...) (14).

Estas dos estrategias para la implantación de las técnicas farmacogenéticas tienen ventajas e inconvenientes desde el punto de vista práctico, ético y económico, dependientes de la situación de cada centro o sistema de salud. En este momento la estrategia “caso-a-caso" es claramente la adoptada mayoritariamente en la práctica clínica (v.gr, abacavir). Sin embargo, la situación está cambiando de manera rápida conforme se introducen las técnicas de genotipado amplio (Arrays, por ejemplo) y la secuenciación masiva. El coste de estas técnicas es cada vez más bajo y se acerca cada vez más al coste del genotipado dirigido, con la ventaja de disponer de la información cuando se necesita y de la obtención de una mayor cantidad de información relevante (actual y futura) para el paciente.

Tabla1. Fármacos para los que existe una recomendación por parte del Clinical Pharmacogenetics Implementation Consortium (CPIC), el Dutch Pharmacogenetics Working Group (DPWG) o ambos.

\begin{tabular}{|c|c|c|}
\hline Ambos & Solo CPIC & Solo DPWC \\
\hline abacavir & allopurinol & aripiprazole \\
\hline amitriptyline & atazanavir & atomoxetine \\
\hline azathioprine & carbamazepine & carvedilol \\
\hline capecitabine & desipramine & clozapine \\
\hline citalopram & fluvoxamine & esomeprazole \\
\hline clomipramine & ivacaftor & flecainide \\
\hline clopidogrel & peginterferon alfa- $2 a$ & flupenthixol \\
\hline codeine & peginterferon alfa- $2 b$ & gliclazide \\
\hline doxepin & rasburicase & glimepiride \\
\hline escitalopram & simvastatin & haloperidol \\
\hline fluorouracil & trimipramine & irinotecan \\
\hline imipramine & warfarin & lansoprazole \\
\hline mercaptopurine & & metoprolol \\
\hline nortriptyline & & mirtazapine \\
\hline paroxetine & & moclobemide \\
\hline phenytoin & & propafenone \\
\hline ribavirin & & olanzapine \\
\hline sertraline & & omeprazole \\
\hline tacrolimus & & oxycodone \\
\hline tegafur & & pantoprazole \\
\hline \multirow[t]{9}{*}{ thioguanine } & & phenprocoumon \\
\hline & & rabeprazole \\
\hline & & risperidone \\
\hline & & tamoxifen \\
\hline & & tolbutamide \\
\hline & & tramadol \\
\hline & & venlafaxine \\
\hline & & voriconazole \\
\hline & & zuclopenthixol \\
\hline
\end{tabular}

Al contrario de la iniciativa eMERGE-PGx, que pretende una implementación en numerosos centros que ya utilizan de forma amplia la información farmacogenética en la práctica clínica, el uso clínico de la técnicas farmacogenéticas en España parece mucho más limitado y la aproximación de un genotipado 
prácticamente universal parece más lejana en nuestro medio. Sin embargo, ante la clara expectativa de que la obtención de la información genética será cada vez más barata y accesible, nuestro grupo plantea evaluar la viabilidad del genotipado anticipado para una serie de patologías concretas, o "poblaciones de riesgo" para las cuales la información genética pueda ser de utilidad de acuerdo a la información, recomendaciones y guías previamente mencionadas. Esta estrategia permitirá aplicar la información genética de manera inmediata en aquellas situaciones con más evidencias e ir incorporando nuevos marcadores conforme se van generando las evidencias.

Así pues, nuestra expectativa es que en un futuro inmediato la implementación de la farmacogenética avance hacia la estrategia del genotipado anticipado en "poblaciones de riesgo", dadas las ventajas que se han mencionado.

\section{REFERENCI AS}

[1] Caudle KE, Klein TE, Hoffman JM et al. Incorporation of pharmacogenomics into routine clinical practice: the Clinical Pharmacogenetics Implementation Consortium (CPIC) guideline development process. Curr Drug Metab. 2014 Feb;15(2):209-17.

[2] Martin MA, Klein TE, Dong BJ, Pirmohamed M, Haas DW, Kroetz DL. Clinical pharmacogenetics implementation consortium guidelines for HLA-B genotype and abacavir dosing. Clin Pharmacol Ther 2012; 91:734-738.

[3] Relling MV, Gardner EE, Sandborn WJ et al. Clinical Pharmacogenetics Implementation Consortium guidelines for thiopurine methyltransferase genotype and thiopurine dosing. Clin Pharmacol Ther 2011; 89:387-391.

[4] Scott SA, Sangkuhl K, Gardner EE et al. Clinical Pharmacogenetics Implementation Consortium guidelines for cytochrome P450-2C19 (CYP2C19) genotype and clopidogrel therapy. Clin Pharmacol Ther 2011; 90:328-332.

[5] Borobia AM, Lubomirov R, Ramirez E et al. An acenocoumarol dosing algorithm using clinical and pharmacogenetic data in Spanish patients with thromboembolic disease. PLoS One 2012; 7:e41360.

[6] Tong HY, Dávila-Fajardo CL, Borobia AM et al. (2016) A New Pharmacogenetic Algorithm to Predict the Most Appropriate Dosage of Acenocoumarol for Stable Anticoagulation in a Mixed Spanish Population. PLoS ONE 11(3): e0150456. doi:10.1371/journal.pone.0150456

[7] Wilke RA, Ramsey LB, Johnson SG et al. The clinical pharmacogenomics implementation consortium: CPIC guideline for SLCO1B1 and simvastatin-induced myopathy. Clin Pharmacol Ther 2012; 92:112-117.

[8] Roberts, J.D. et al. Point-of-care genetic testing for personalisation of antiplatelet treatment (RAPID GENE): a prospective, randomised, proof-ofconcept trial. Lancet. 2012 May 5;379(9827):1705-11

[9] Johnson JA, Burkley BM, Langaee TY et al. Implementing personalized medicine: development of a cost-effective customized pharmacogenetics genotyping array. Clin Pharmacol Ther 2012; 92:437-439

[10] Crews KR, Hicks JK, Relling MV and Evans WE. Pharmacogenomics and Individualized Medicine: Translating Science Into Practice. Clin Pharmacol Ther 2012; 92:467-75

[11] Pulley, J.M. et al. Operational implementation of prospective genotyping for personalized medicine: the design of the Vanderbilt PREDICT project. Clin. Pharmacol. Ther 2012; 92:87 -95;

[12] Bielinski SJ et al. Preemptive genotyping for personalized medicine: design of the right drug, right dose, right timeusing genomic data to individualize treatment protocol. Mayo Clin Proc. 2014 Jan;89(1):2533

[13] Rasmussen-Torvik LJ, Stallings SC, Gordon AS et al. Design and anticipated outcomes of the eMERGE-PGx project: a multicenter pilot for preemptive pharmacogenomics in electronic health record systems. Clin Pharmacol Ther. 2014 Oct;96(4):482-9. doi: 10.1038/clpt.2014.137.

[14] Borobia A.M., Lubomirov R., Tong H.Y. et al. Implementing pharmacogenetics: Pharmarray, Acodose and pharmacogenetic consultation. Basic \& Clinical Pharmacology \& Toxicology, 2013;113(Suppl.2),16 37. 